\title{
Quantum reflection of antihydrogen from a liquid helium film
}

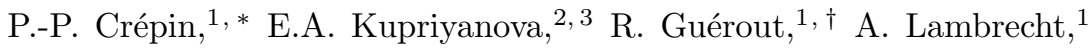 \\ V.V. Nesvizhevsky, ${ }^{4}$ S. Reynaud, ${ }^{1,}$ f $\mathrm{S}$. Vasiliev, ${ }^{5}$ and A.Yu. Voronin ${ }^{2,3}$ \\ ${ }^{1}$ Laboratoire Kastler Brossel (LKB), UPMC-Sorbonne Universités, CNRS, \\ ENS-PSL Research University, Collège de France, 75252, Paris, France \\ ${ }^{2}$ P.N. Lebedev Physical Institute, 53 Leninsky prospect, 117924, Moscow, Russia \\ ${ }^{3}$ Russian Quantum Center, 100 A, Novaya street, Skolkovo, 143025, Moscow, Russia \\ ${ }^{4}$ Institut Laue-Langevin (ILL), 71 avenue des Martyrs, 38042, Grenoble, France \\ ${ }^{5}$ Department of Physics and Astronomy, University of Turku, 20014, Turku, Finland
}

(Dated: March 13, 2018)

\begin{abstract}
We study the quantum reflection of ultracold antihydrogen atoms bouncing on the surface of a liquid helium film. The Casimir-Polder potential and quantum reflection are calculated for different thicknesses of the film supported by different substrates. Antihydrogen can be protected from annihilation for as long as $1.3 \mathrm{~s}$ on a bulk of liquid ${ }^{4} \mathrm{He}$, and $1.7 \mathrm{~s}$ for liquid ${ }^{3} \mathrm{He}$. These large lifetimes open interesting perspectives for spectroscopic measurements of the free fall acceleration of antihydrogen. Variation of the scattering length with the thickness of a film of helium shows interferences which we interpret through a Liouville transformation of the quantum reflection problem.
\end{abstract}

\section{INTRODUCTION}

Quantum reflection is a non classical phenomenon which appears when a quantum matter wave approaches a rapidly varying attractive potential. Instead of accelerating towards the surface, the quantum particle has a probability to be reflected. This process has been studied theoretically for the van der Waals potential since the early days of quantum mechanics [1-4]. It was first observed experimentally for $\mathrm{H}$ and He atoms [5] 7] and then for ultracold atoms or molecules on solid surfaces 8 [11].

In the last years quantum reflection has been studied also for antimatter $12-14$ since it should play a key role in experiments with antihydrogen atoms [15-17. It was shown that the free fall acceleration of antihydrogen can in principle be evaluated accurately [18, through spectroscopic studies of the quantum levitational states [19, 20] of atoms trapped by quantum reflection and gravity [21, 22. Following uncertainty principle of quantum mechanics, such spectroscopic measurements should have a better accuracy for larger lifetime of antihydrogen in the trap.

In this letter, we calculate quantum reflection of antihydrogen above liquid helium films and show that the lifetime of antihydrogen reaches values as high as $1.3 \mathrm{~s}$ for a bulk (a thick film) of liquid ${ }^{4} \mathrm{He}$ and $1.7 \mathrm{~s}$ for a bulk of liquid ${ }^{3} \mathrm{He}$. We also study the effect of thickness for ${ }^{4} \mathrm{He}$ films supported by different substrates and bring out a surprising interference pattern for the scattering length as a function of thickness. By using a Liouville transformation of the quantum reflection problem, we propose an interpretation of this phenomenon in terms of shape resonances.

\section{CASIMIR-POLDER POTENTIAL}

We study quantum reflection for an antihydrogen atom of mass $m$ falling onto a liquid helium film of thickness $d$ supported by a substrate (see Fig. 1).

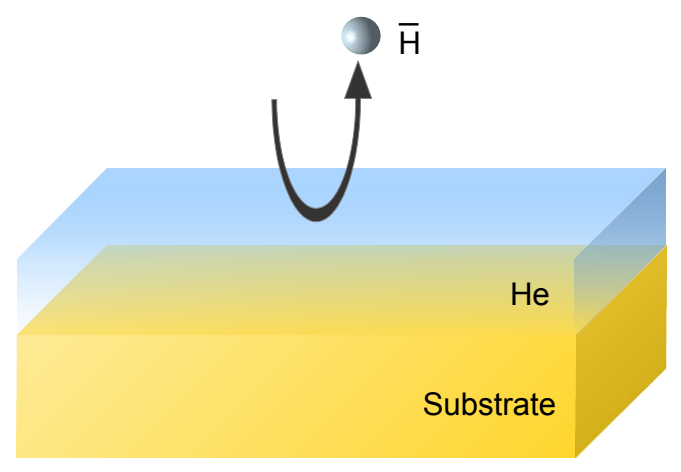

Figure 1. Representation of the quantum reflection process for an antihydrogen atom falling onto a helium film supported by a substrate. We study the limiting case of a bulk of helium (very large thickness of the film) as well as the general case of a film of finte thickness $d$ supported by a substrate.

The atom is sensitive to the Casimir-Polder (CP) potential and to the free fall acceleration $\bar{g}$. In the present letter, we focus our attention on the quantum reflection in the CP potential [23], as the latter is effective at distances $z$ much smaller than the length scale $\ell_{g}$ associated with quantum effects in the gravity field $\ell_{g}=\left(\hbar^{2} / 2 m^{2} g\right)^{1 / 3} \simeq 5.87 \mu \mathrm{m}$. Note that we suppose $\bar{g}=g$ in all numerical evaluations.

The CP potential is evaluated at zero temperature as the following function of the distance $z$ of the atom to 
the liquid helium surface 24]

$$
\begin{aligned}
& V(z)=\frac{\hbar}{c^{2}} \int_{0}^{\infty} \frac{d \xi}{2 \pi} \xi^{2} \alpha(i \xi) \int \frac{d^{2} \mathbf{q}}{(2 \pi)^{2}} \frac{e^{-2 \kappa z}}{2 \kappa} \sum_{p} s_{p} r_{p}, \\
& \kappa=\sqrt{\xi^{2} / c^{2}+\mathbf{q}^{2}}, \quad s_{\mathrm{TE}}=1, \quad s_{\mathrm{TM}}=-\frac{\xi^{2}+2 c^{2} \mathbf{q}^{2}}{\xi^{2}} .
\end{aligned}
$$

This expression is integrated over the complex frequency $\omega=i \xi$ and the transverse wave-vector $\mathbf{q}$, and summed up over the two field polarizations $p=\mathrm{TE}$, TM.

The dynamic polarizability $\alpha(\omega)$ of the antihydrogen atom is supposed to be the same as for the hydrogen atom, because possible differences between the two cases would be too small to have an influence at the level of precision aimed in the present study. Note that there are differences between hydrogen and antihydrogen for the atomic physics in the proximity of the helium film. While the potential of interaction for a hydrogen atom leads to the existence of a single bound state with an adsorption energy of the order of $1 \mathrm{~K}$ on ${ }^{4} \mathrm{He}$ bulk [25 27], the repulsive part of potential may be absent in the much less studied case of antihydrogen. These differences are not studied in this letter where we focus attention on antihydrogen for which quantum reflection is the only reason for scattering of atoms back from the helium surface.

The reflection amplitudes $r_{p}$ are calculated for polarizations $p$ by combining the Fresnel amplitudes at interfaces and propagation in the helium film. The optical properties of ${ }^{4} \mathrm{He}$ are described with a sufficient accuracy by a model dielectric constant with three resonances 28

$$
\begin{aligned}
& \epsilon(i \xi) \simeq 1+\sum_{k=1,2,3} \frac{a_{k}}{1+\left(\xi / \omega_{k}\right)^{2}} \\
& \left(\omega_{1}, \omega_{2}, \omega_{3}\right)=(3.22,3.74,12) \times 10^{16}{\mathrm{rad} . \mathrm{s}^{-1}} \\
& \left(a_{1}, a_{2}, a_{3}\right)=(0.016,0.036,0.0047)
\end{aligned}
$$

This model corresponds to a dielectric constant close to unity at the static limit $(\epsilon(0)-1 \simeq 0.0567)$ as well as at all frequencies. We also use the optical model (2) for ${ }^{3} \mathrm{He}$, with the same resonance frequencies $\omega_{k}$, and the resonance amplitudes $a_{k}$ multiplied by the same factor calculated to reproduce the static dielectric constant $\epsilon(0)-1 \simeq 0.043$ known from experiments [29].

These numbers lead to a poor reflectance of the film for electromagnetic waves and weak values for the $\mathrm{CP}$ potential with values even weaker for ${ }^{3} \mathrm{He}$ than for ${ }^{4} \mathrm{He}$. It follows that quantum reflection occurs closer to the material surface where the $\mathrm{CP}$ potential is much steeper, which explains the large quantum reflection probability found below, with reflection even larger for ${ }^{3} \mathrm{He}$ than for ${ }^{4} \mathrm{He}$. In both cases we use an effective dielectric constant and disregard the role played by excitations in the helium film like ripplons. The latter is well justified at temperatures below $100 \mathrm{mK}$ 30, the temperature range where results obtained in the following are accurate.
We now discuss the results calculated for the CP potential in different situations. We begin with the limiting cases of $d \rightarrow 0$ and $d \rightarrow \infty$ where we obtain respectively the $\mathrm{CP}$ potentials of the naked substrate and of liquid helium bulk (that is liquid helium film with a large thickness). These potentials, attractive at all distances, behave as non retarded van der Waals potentials at short distances and as retarded potentials at large distances, with the two domains separated by the wavelength $\lambda_{A} \simeq 121 \mathrm{~nm}$ of the first atomic transition $1 \mathrm{~S} \rightarrow 2 \mathrm{P}$ of antihydrogen

$$
\begin{aligned}
& V(z) \simeq-\frac{C_{3}}{z^{3}}, \quad z \ll \lambda_{A}, \\
& V(z) \simeq-\frac{C_{4}}{z^{4}}, \quad z \gg \lambda_{A} .
\end{aligned}
$$

Constants $C_{3}$ and $C_{4}$ are given in table 1 for liquid helium bulk and substrates made of silica, silicon or gold.

\begin{tabular}{|c|c|c|}
\hline medium & $C_{3}\left[\mathrm{E}_{\mathrm{h}} \mathrm{a}_{0}^{3}\right]$ & $C_{4}\left[\mathrm{E}_{\mathrm{h}} \mathrm{a}_{0}^{4}\right]$ \\
\hline \hline liquid ${ }^{3} \mathrm{He}$ & 0.0034 & 1.19 \\
\hline liquid ${ }^{4} \mathrm{He}$ & 0.0045 & 1.55 \\
\hline silica & 0.053 & 28.1 \\
\hline silicon & 0.101 & 50.28 \\
\hline gold & 0.085 & 73.38 \\
\hline
\end{tabular}

Table I. Constants $C_{3}$ and $C_{4}$ for bulks of liquid helium and substrates made of silica, silicon or gold, expressed in atomic units ( $E_{h}$ and $a_{0}$ are the Hartree energy and Bohr radius).

For the purpose of discussing the influence of the optical properties of the mirror on the $\mathrm{CP}$ potentials, we normalize the potential $V(z)$ obtained from (2) by the potential $V_{*}(z)=-C_{4}^{*} / z^{4}$ corresponding to the large distance limit above a perfectly reflecting mirror. The constant $C_{4}^{*}=-3 \alpha(0) \hbar c /\left(32 \pi^{2}\right)$ is determined by the static polarizability $\alpha(0)=\frac{9}{2} \mathrm{a}_{0}^{3}$ of antihydrogen.

The ratios $V(z) / V_{*}(z)$ obtained for liquid ${ }^{3} \mathrm{He}$ and ${ }^{4} \mathrm{He}$ bulks as well as silica, silicon and gold bulks are plotted as full lines in Fig. 2. The ratios obtained for liquid ${ }^{4} \mathrm{He}$ films with finite thickness $d$ on silica are plotted in Fig. 2 as dashed lines. They go smoothly from the one obtained for a liquid helium bulk for $z \ll d$ to that for a silica bulk for $z \gg d$.

\section{QUANTUM REFLECTION FROM A LIQUID HELIUM BULK}

The previous calculations show a very low value of the $\mathrm{CP}$ potential for thick enough liquid helium films, as explained by the fact that liquid helium is almost transparent for the electromagnetic field. We now discuss the consequence of this fact in terms of large quantum reflection from liquid helium bulks.

To this aim, we solve the Schrödinger equation for the antihydrogen falling into the CP potential [15] above the 
liquid helium film. We then obtain the reflection amplitude $r$ as the ratio of the outgoing wave to the incoming one far from the film. The quantum reflection probability is the squared modulus of this amplitude $R=|r|^{2}$. The results are shown in Fig. 3 with larger and larger probability obtained for the weaker and weaker potentials of Fig. 2. In particular, quantum reflection for atoms falling from a height $h$ and thus having a given energy $E=m g h$ is much larger on a liquid helium bulk than on the other materials studied here.

We then extract from the reflection amplitude the complex length $\mathcal{A}(k)$ depending on the wavevector $k$ equivalent to the energy $E=\hbar^{2} k^{2} /(2 m)$

$$
\mathcal{A}(k) \equiv-\frac{i}{k} \frac{1+r(k)}{1-r(k)}
$$

The effective range theory modified to account for the $-C_{4} / z^{4}$ tail of the potential leads to the expression

$$
\begin{aligned}
\mathcal{A}(k) & =-i \ell \alpha(k \ell), \\
\alpha(K) & =\alpha_{0}+i \frac{\pi}{3} K+\left(\alpha_{2}+\frac{4}{3} \alpha_{0} \ln K\right) K^{2},
\end{aligned}
$$

where $\ell=\sqrt{2 m C_{4}} / \hbar$ is the characteristic length associated with the constant $C_{4}$ while $\alpha_{0}$ and $\alpha_{2}$ are two dimensionless parameters. The values of $\alpha_{0}$ and $\alpha_{2}$ are known for the special case $V=-C_{4} / z^{4}$ [31, 32. In the problem studied here, the $\mathrm{CP}$ potential above the liquid helium film does not reduce to this special form, and we proceed as in [18] by extracting the 2 parameters from a fit of the low $-k$ dependence of $r(k)$.

We now discuss the results thus obtained for the scattering length $a=-i \ell \alpha_{0}$ which is deduced from the characteristic length $\ell$ and the parameter $\alpha_{0}$. For quantum reflection on a liquid ${ }^{4} \mathrm{He}$ bulk, one finds for example $a=-(34.983+44.837 i) a_{0}$. The imaginary part

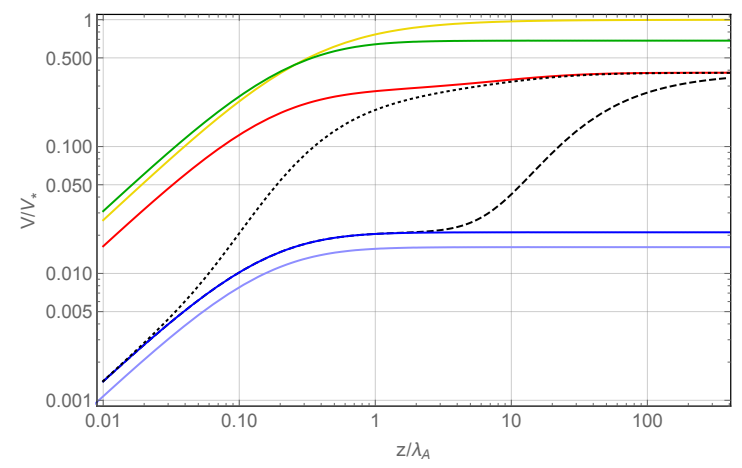

Figure 2. CP potentials $V(z)$ normalized by the potential $V_{*}(z)$ calculated for a perfect mirror at large distances. Distances $z$ are normalized by the wavelength $\lambda_{A} \simeq 121 \mathrm{~nm}$ of the $1 \mathrm{~S} \rightarrow 2 \mathrm{P}$ antihydrogen transition. The full lines correspond, from bottom to top, to bulks of ${ }^{3} \mathrm{He}$ (light blue), ${ }^{4} \mathrm{He}$ (dark blue), silica (red), silicon (green) and gold (yellow). The other lines correspond to liquid helium films of thickness $d=10 \lambda_{A}$ (dashed line) and $d=0.1 \lambda_{A}$ (dotted line) on a silica bulk.

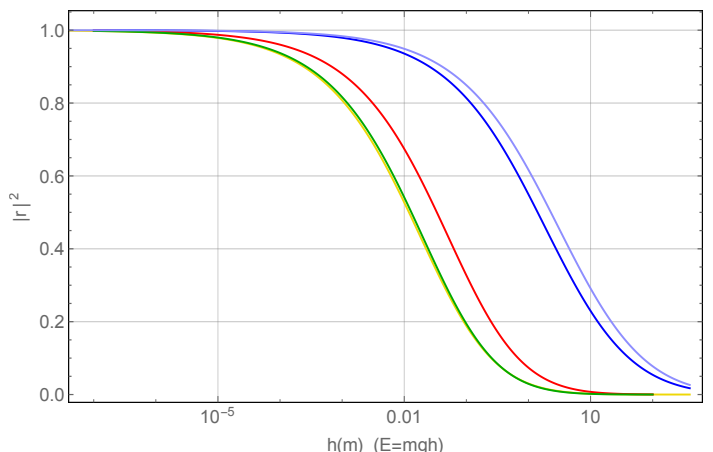

Figure 3. Quantum reflection probability as a function of the free fall height of the atom $h$, that is also of its energy $E=m g h$. The full lines correspond, from top to bottom, to bulks of ${ }^{3} \mathrm{He}$ (light blue), ${ }^{4} \mathrm{He}$ (dark blue), silica (red), silicon (green) and gold (yellow).

$b=-\operatorname{Im}(a)$ of this scattering length determines the mean lifetime $\tau$ for atoms bouncing above the bulk [18]

$$
\tau=\frac{\hbar}{2 m g b} .
$$

In table II] we compare this values obtained for $\tau$ from the quantum reflection propabilities drawn on Fig. 3 and also for porous silica studied in [16. We also give the values for the number $N_{1}$ of bounces for an atom in the first quantum levitation state. The numbers show that liquid helium is a much better reflector for antihydrogen matter waves than the other materials which have been studied up to now. The much larger lifetime, that is also the much larger number of bounces before annihilation, implies that it should be possible to trap antimatter for long enough to improve significantly the spectroscopy measurements discussed in [18.

\begin{tabular}{|c|c|c|}
\hline material & $\tau[\mathrm{s}]$ & $N_{1}$ \\
\hline \hline perfectly reflective & 0.11 & 33 \\
\hline silica bulk & 0.22 & 66 \\
\hline porous silica (95\% porosity) & 0.94 & 282 \\
\hline liquid ${ }^{4} \mathrm{He}$ bulk & 1.35 & 405 \\
\hline liquid ${ }^{3} \mathrm{He}$ bulk & 1.71 & 514 \\
\hline
\end{tabular}

Table II. Lifetime $\tau$ of antihydrogen in seconds above various material surfaces and number $N_{1}$ of bounces for an atom in the first quantum gravitational state for different bulk materials and for porous silica (see [16] for the latter case).

\section{QUANTUM REFLECTION FROM FINITE THICKNESS FILMS}

We now investigate the effect on quantum reflection of the finite thickness of a liquid ${ }^{4} \mathrm{He}$ film supported by a substrate. We present the results of the calculations 
in terms of the scattering length $a$ which now depends on the thickness $d$ of the film as well as on the optical properties of the substrate.
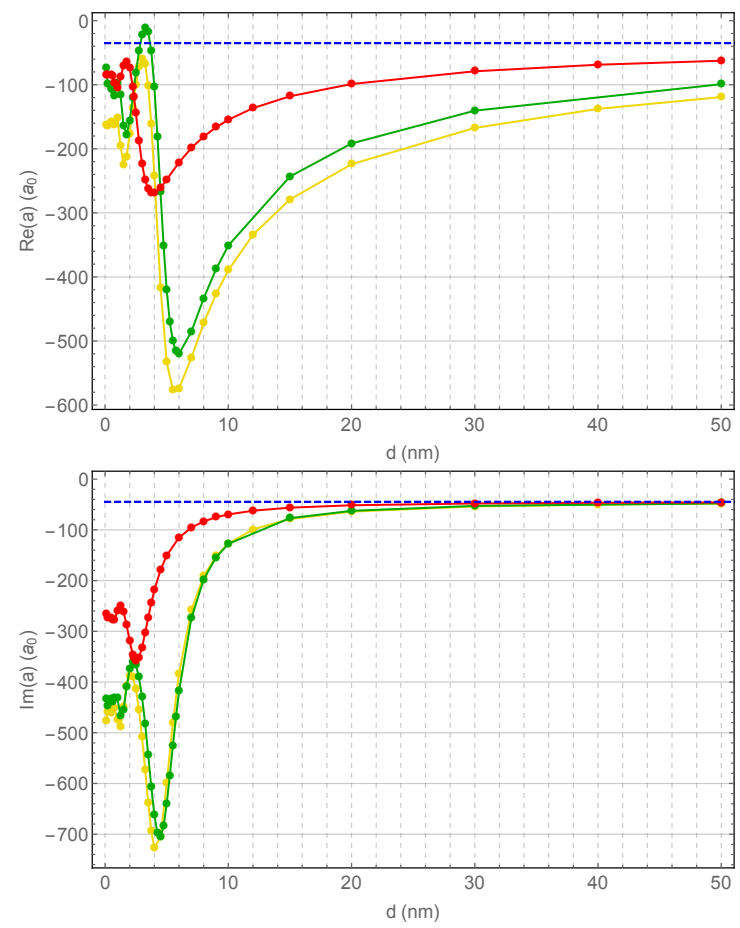

Figure 4. Real (upper plot) and imaginary (lower plot) parts of the scattering length depending on the thickness $d$ of the liquid ${ }^{4} \mathrm{He}$ film, drawn from the top to the bottom for a silica substrate (red curve), a silicon substrate (green) and a gold substrate (yellow). For comparison, the dashed (blue) line corresponds to real and imaginary parts of the scattering length for a liquid ${ }^{4} \mathrm{He}$ bulk.

Results are presented in Fig. 4 for films supported by silica, silicon and gold substrates. The important effect of the thickness is clearly seen on this plot. For thicknesses larger than a few tens of nanometers, the scattering length reaches asymptotically the value found above for a liquid ${ }^{4} \mathrm{He}$ bulk. The curves give the thickness of the film to be chosen sufficient for recovering the large lifetimes predicted at the limit of the bulk. This property is also illustrated in terms of variation of the lifetime in Fig. 5. As could be expected, the substrate which leads to the larger lifetime for a given thickness of the liquid helium film is the one which would have the best reflectivity without the film (silica in our case).

For small thicknesses, of the order of a few nanometers, real and imaginary parts of the scattering length are found to oscillate in phase quadrature in Fig. 44.5. This property is confirmed by the variation of $a$ in the complex plane, shown in Fig. 6 in the case of a gold substrate. It looks like a consequence of an interference phenomenon that we discuss in the next section.

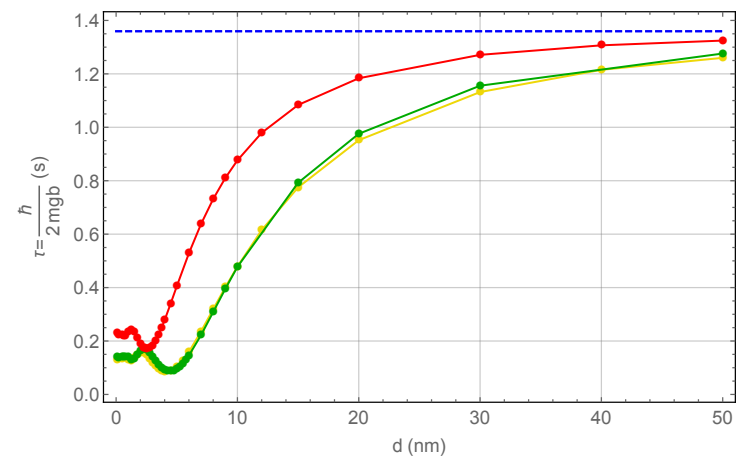

Figure 5. Lifetime $\tau$ depending on the thickness $d$ of the liquid ${ }^{4} \mathrm{He}$ film, drawn from the top to the bottom for a silica substrate (red curve), a silicon substrate (green) and a gold substrate (yellow). For comparison, the dashed (blue) line is the lifetime corresponding to the liquid ${ }^{4} \mathrm{He}$ bulk.

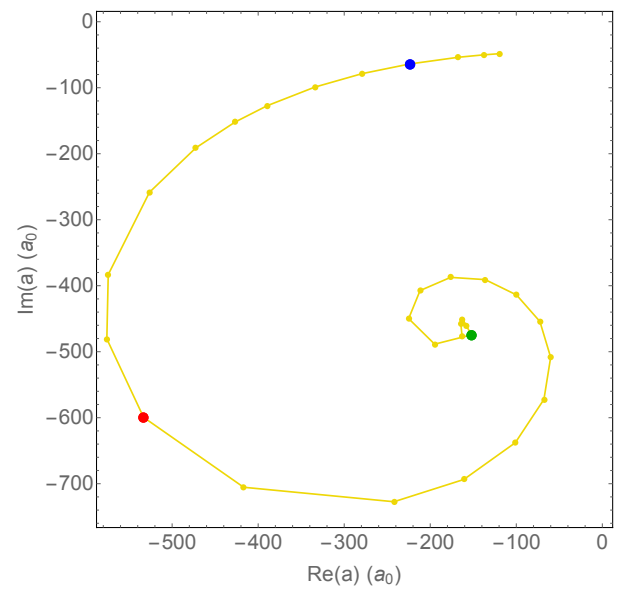

Figure 6. Scattering length a represented in the complex plane, depending on the thickness $d$ of the liquid helium film above a gold substrate. The thickness ranges from $0.1 \mathrm{~nm}$ (center of the spiral) to $50 \mathrm{~nm}$ (outer part of the spiral). Green point corresponds to $d=1 \mathrm{~nm}$, red to $d=5 \mathrm{~nm}$ and blue to $d=20 \mathrm{~nm}$.

\section{DISCUSSION OF THE OSCILLATIONS}

The counterintuitive property of a larger quantum reflection for a weaker CP potential [15, 16] has been discussed in recent papers by using Liouville transformations of the Schrödinger equation. Such transformations allow one to map the problem of quantum reflection from an atractive well to the more intuitive problem of ordinary reflection from a wall, with the latter becoming higher for weaker CP potentials [33, 34].

The shape of the CP potential for the film of thickness $10 \lambda_{A}$ in Fig. 2 suggests that the atom falling onto the film see two zones of rapid variation of the potential, the first one at the transition from the potential which would be seen for the naked substrate to that of a helium bulk, and the second one at the approach to the liquid helium 
film. Using Liouville transformations, we now interpret the oscillations of the scattering length seen in Fig. 6] as an interference between reflections on the two walls.

Liouville transformations [35, 36] are gauge transformations of the Schrödinger equation which preserve the reflection amplitude while changing the potential landscape. They correspond to a coordinate change $z \rightarrow \boldsymbol{z}$ and rescaling $\psi \rightarrow \boldsymbol{\psi}=\sqrt{\boldsymbol{z}^{\prime}(z)} \psi$ of the wave-function We choose here a specific Liouville gauge discussed in [34, with the new coordinate $\boldsymbol{z}=\phi_{\mathrm{dB}} / \varkappa$ proportional to the WKB phase $\phi_{\mathrm{dB}}(\varkappa$ an arbitrary constant). The latter is the integral $\phi_{\mathrm{dB}}=\int k_{\mathrm{dB}} d z$ of the WKB wavevector $k_{\mathrm{dB}}=\sqrt{2 m(E-V)} / \hbar$. The Liouville transformation preserves the form of the Schrödinger equation with modified energy $\boldsymbol{E}$ and potential $\boldsymbol{V} 34$

$$
\begin{aligned}
& \boldsymbol{V}(\boldsymbol{z})=\boldsymbol{E} Q(z) \quad, \quad \boldsymbol{E}=\varkappa^{2} \\
& Q(z)=-\alpha_{\mathrm{dB}}^{3} \frac{d^{2} \alpha_{\mathrm{dB}}}{d z^{2}} \quad, \quad \alpha_{\mathrm{dB}} \equiv \frac{1}{\sqrt{k_{\mathrm{dB}}}} .
\end{aligned}
$$

$Q(z)$ is the badlands function [3, 4, which marks the zones where the WKB approximation breaks down, that is also where significant quantum reflection occurs [15].

We draw in Fig. 7 the badlands function $Q(z)$ for four different thicknesses of the film supported by a gold substrate. Three thicknesses correspond to the colored points emphasized in Fig. 4 for $d=1 \mathrm{~nm}$ (green), $5 \mathrm{~nm}$ (red) and 20nm (blue). For the purpose of comparison with the case of a naked substrate, a fourth plot is drawn for $d=0$ (dashed yellow curve). The plots for non null thicknesses show two peaks while only one peak appears for the plot of the naked substrate as well as for the plot of a liquid helium bulk. The peak lying far from the surface is roughly the same for all curves, and it is the same as for the naked substrate. The other one corresponds to the approach to the helium film and its position depends on the thickness of the film.

For films with non null thicknesses, the two peaks form a cavity where the matter wave can be stored. In the case studied here, the mirror closer to the material surface has a poorer reflectivity than the mirror farther from the material surface. The presence of the cavity leads to a faster annihilation when atoms are trapped, which degrades the lifetime of the antihydrogen atom, as observed in Fig. 4 5. The interferences taking place in the cavity explain the oscillation patterns highlighted in Fig. 4 and 6 . The associated phase is related to the round-trip dephasing in the cavity, which is determined by the displacement to the left of the weaker peak and the change of the shape of the potential inside the cavity. This discussion can be considered as a qualitative interpretation of the full calculations presented in the preceding section.

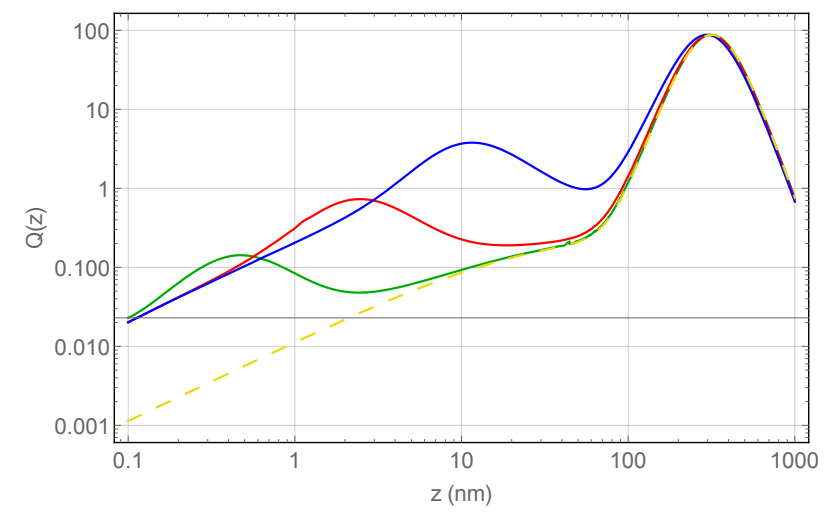

Figure 7. Badlands functions $Q(z)$ ( $z$ in $\mathrm{nm}$ ) calculated for an antihydrogen atom falling from the energy of the first quantum gravitational state onto a liquid helium film above a gold substrate. The three full lines correspond to three thicknesses of the film, with the same color code as for the points emphasized in Fig. 6 : From bottom to top, the thickness of the film is $1 \mathrm{~nm}$ (green), $5 \mathrm{~nm}$ (red) and $20 \mathrm{~nm}$ (blue). The dashed (yellow) curve corresponds to the naked substrate.

\section{CONCLUSION}

In this letter we have found theoretically a high reflection probability for antihydrogen atoms falling down onto thick enough liquid helium films. We also predicted the presence of oscillations of the scattering length as a function of thickness for liquid helium films supported by a substrate. We interpreted the associated interference pattern as a consequence of the existence of two separated zones where significant reflection occurs.

We have considered that low-temperature reflection properties of antihydrogen atoms from liquid helium films are essentially determined by the long-range part of potential, as it is known theoretically [37] and proven experimentally [7] for the case of hydrogen-liquid helium interaction. Of course, it would be interesting to go further in the analysis by considering explicitly the effect of the short-range part of the interaction potential. This idea has been studied for the case of antihydrogen atom-helium atom interaction, and short-range repulsion predicted 38,42$]$. It would be worth performing similar calculations for the case of antihydrogen atom-liquid helium interaction studied in the present letter. These calculations may change the precise value of the lifetimes obtained above, but they should not affect qualitatively our main statement, namely that long lifetimes are obtained for antimatter above a liquid helium bulk, which have very interesting applications for new spectroscopic tests of the equivalence principle for antihydrogen atoms.

Acknowledgements - We are grateful to our colleagues from GBAR and GRANIT collaborations for useful discussions. We thank the referee for stimulating comments. 
* pierre-philippe.crepin@lkb.upmc.fr

† romain.guerout@lkb.upmc.fr

‡ serge.reynaud@lkb.upmc.fr

[1] J. E. Lennard-Jones and A. F. Devonshire. The interaction of atoms and molecules with solid surfaces. III. The Condensation and Evaporation of Atoms and Molecules. Proceedings of the Royal Society of London. Series A, 156:6-28, 1936.

[2] J. E. Lennard-Jones and A. F. Devonshire. The interaction of atoms and molecules with solid surfaces. IV. The condensation and evaporation of atoms and molecules. Proceedings of the Royal Society of London. Series A, 156:29-36, 1936.

[3] M. V. Berry and K. E. Mount. Semiclassical approximations in wave mechanics. Reports on Progress in Physics, 35:315-397, 1972.

[4] H. Friedrich and J. Trost. Working with WKB waves far from the semiclassical limit. Physics Reports, 397:359449, 2004.

[5] V. U. Nayak, D. O. Edwards, and N. Masuhara. Scattering of ${ }^{4} \mathrm{He}$ Atoms Grazing the Liquid- ${ }^{4} \mathrm{He}$ Surface. Physical Review Letters, 50:990-992, 1983.

[6] J. J. Berkhout, O. J. Luiten, I. D. Setija, T. W. Hijmans, T. Mizusaki, and J. T. M. Walraven. Quantum reflection: Focusing of hydrogen atoms with a concave mirror. Physical Review Letters, 63:1689-1692, 1989.

[7] I. A. Yu, J. M. Doyle, J. C. Sandberg, C. L. Cesar, D. Kleppner, and T. J. Greytak. Evidence for universal quantum reflection of hydrogen from liquid ${ }^{4} \mathrm{He}$. Physical Review Letters, 71:1589-1592, 1993.

[8] F. Shimizu. Specular reflection of very slow metastable neon atoms from a solid surface. Physical Review Letters, 86:987-990, 2001.

[9] V. Druzhinina and M. DeKieviet. Experimental observation of quantum reflection far from threshold. Physical Review Letters, 91:193202, 2003.

[10] T. A. Pasquini, Y. Shin, C. Sanner, M. Saba, A. Schirotzek, D. E. Pritchard, and W. Ketterle. Quantum reflection from a solid surface at normal incidence. Physical Review Letters, 93:223201, 2004.

[11] T. A. Pasquini, M. Saba, G.-B. Jo, Y. Shin, W. Ketterle, D. E. Pritchard, T. A. Savas, and N. Mulders. Low velocity quantum reflection of Bose-Einstein condensates. Physical Review Letters, 97:093201, 2006.

[12] A. Y. Voronin, P. Froelich, and B. Zygelman. Interaction of ultracold antihydrogen with a conducting wall. Physical Review A, 72:062903, 2005.

[13] A. Yu Voronin, P. Froelich, and V. V. Nesvizhevsky. Gravitational quantum states of Antihydrogen. Physical Review A, 83:032903, 2011.

[14] A. Yu Voronin, V. V. Nesvizhevsky, and S. Reynaud. Whispering-gallery states of antihydrogen near a curved surface. Physical Review A, 85:014902, 2012.

[15] G. Dufour, A. Gérardin, R. Guérout, A. Lambrecht, V. V. Nesvizhevsky, S. Reynaud, and A. Yu. Voronin. Quantum reflection of antihydrogen from the Casimir potential above matter slabs. Physical Review A, 87:012901, 2013.

[16] G. Dufour, R. Guérout, A. Lambrecht, V. V. Nesvizhevsky, S. Reynaud, and A. Yu. Voronin. Quantum reflection of antihydrogen from nanoporous media.
Physical Review A, 87:022506, 2013.

[17] G. Dufour, P. Debu, A. Lambrecht, V. V. Nesvizhevsky, S. Reynaud, and A. Yu Voronin. Shaping the distribution of vertical velocities of antihydrogen in GBAR. European Physical Journal C, 74:2731, 2014.

[18] P.-P. Crépin, G. Dufour, R. Guérout, A. Lambrecht, and S. Reynaud. Casimir-Polder shifts on quantum levitation states. Physical Review A, 95:032501, 2017.

[19] G. Breit. The propagation of Schrödinger waves in a uniform field of force. Phys. Rev., 32:273-276, 1928.

[20] V. V. Nesvizhevsky, H. G. Börner, A. K. Petukhov, H. Abele, S. Baeßler, F. J. Rueß, T. Stöferle, A. Westphal, A. M. Gagarski, G. A. Petrov, and A. V. Strelkov. Quantum states of neutrons in the Earth's gravitational field. Nature, 415:297-299, 2002.

[21] A. Jurisch and H. Friedrich. Realistic model for a quantum reflection trap. Physics Letters A, 349:230-235, 2006.

[22] J. Madroñero and H. Friedrich. Influence of realistic atom wall potentials in quantum reflection traps. Physical Review A, 75:022902, 2007.

[23] The full problem of combined effects of the CP and gravity potentials is treated in 18 .

[24] R. Messina, D. A. R. Dalvit, P. A. Maia Neto, A. Lambrecht, and S. Reynaud. Dispersive interactions between atoms and nonplanar surfaces. Physical Review A, 80:022119, 2009.

[25] B. Castaing and M. Papoular. Kapitza resistance at the $\mathrm{H} /$ liquid He interface. Journal de Physique Lettres, 44:537-540, 1983.

[26] Yu. Kagan, G. V. Shlyapnikov, and N. A. Glukhov. Kapitsa jump in a gas of spin-polarized atomic hydrogen. JETP Letters, 40:1052, 1984.

[27] J. J. Berkhout and J. T. M. Walraven. Scattering of hydrogen atoms from liquid-helium surfaces. Physical Review B, 47:8886-8904, 1993.

[28] E. S. Sabisky and C. H. Anderson. Verification of the Lifschitz theory of the van der Waals potential using liquid helium films. Phys. Rev. A, 7:790, 1972.

[29] H. A. Kierstead. Dielectric constant and molar volume of saturated liquid $3 \mathrm{He}$ and $4 \mathrm{He}$. Journal of Low Temparature Physics, 23:791, 1976.

[30] V. V. Goldman. Kapitza conductance between gaseous atomic hydrogen and liquid helium. Physical Review Letters, 56:612-615, 1986.

[31] T. F. O'Malley, L. Spruch, and L. Rosenberg. Modification of effective-range theory in the presence of a longrange $r^{-4}$ potential. Journal of Mathematical Physics, 2:491-498, 1961.

[32] F. Arnecke, H. Friedrich, and J. Madroñero. Effectiverange theory for quantum reflection amplitudes. Physical Review A, 74:062702, 2006.

[33] G. Dufour, R. Guérout, A. Lambrecht, and S. Reynaud. Quantum reflection and Liouville transformations from wells to walls. EPL (Europhysics Letters), 110:30007, 2015.

[34] G. Dufour., R. Guérout, A. Lambrecht, and S. Reynaud. Liouville transformations and quantum reflection. Journal of Physics B, 48:155002, 2015.

[35] J. Liouville. Second mémoire sur le développement des fonctions ou parties de fonctions en séries dont les divers termes sont assujétis à satisfaire à une même équation différentielle du second ordre, contenant un paramètre variable. Journal de Mathématiques Pures et Appliquées, 
$2: 16-35,1837$.

[36] F. Olver. Asymptotics and special functions. Taylor \& Francis, January 1997.

[37] C. Carraro and M. W. Cole. Role of long-range forces in H sticking to liquid He. Physical Review B, 45:1293012935, 1992.

[38] K. Strasburger and H. Chojnacki. Helium-antihydrogen interaction: The Born-Oppenheimer potential energy curve. Physical Review Letters, 88:163201, 2002.

[39] S. Jonsell, P. Froelich, S. Eriksson, and K. Strasburger. Strong nuclear force in cold antihydrogen-helium collisions. Physical Review A, 70:062708, 2004.
[40] K. Strasburger, H. Chojnacki, and A. Sokolowska. Adiabatic potentials for the interaction of atomic antihydrogen with $\mathrm{He}$ and $\mathrm{He}^{+}$. Journal of Physics B, 38:3091, 2005.

[41] P. Froelich and A. Y. Voronin. Interaction of antihydrogen with ordinary atoms and solid surfaces. Hyperfine Interactions, 213:115-127, 2012.

[42] S Jonsell, E A G Armour, M Plummer, Y Liu, and A C Todd. Helium-antihydrogen scattering at low energies. New Journal of Physics, 14:035013, 2012. 